BI-TP 2002/11

November 2002

cond-mat/0209492 v2

\title{
Numerical equation of state and other scaling functions from an improved three-dimensional Ising model
}

\author{
J. Engels, L. Fromme and M. Seniuch \\ Fakultät für Physik, Universität Bielefeld, D-33615 Bielefeld, Germany
}

\begin{abstract}
We study an improved three-dimensional Ising model with external magnetic field near the critical point by Monte Carlo simulations. From our data we determine numerically the universal scaling functions of the magnetization, that is the equation of state, of the susceptibility and of the correlation length. In order to normalize the scaling functions we calculate the critical amplitudes of the three observables on the critical line, the phase boundary and the critical isochore. The amplitudes lead to the universal ratios $C^{+} / C^{-}=4.756(28), R_{\chi}=1.723(13), Q_{c}=0.326(3)$ and $Q_{2}=1.201(10)$. We find excellent agreement of the data with the parametric representation of the asymptotic equation of state as found by field theory methods. The comparison of the susceptibility data to the corresponding scaling function shows a marginal difference in the symmetric phase, which can be explained by the slightly different value for $R_{\chi}$ used in the parametrization. The shape of the correlationlength-scaling function is similar to the one of the susceptibility, as expected from earlier parametrizations. The peak positions of the two scaling functions are coinciding within the error bars.
\end{abstract}

PACS : 64.10.+h; 75.10.Hk; 05.50+q

Keywords: Ising model; Equation of state; Scaling function; Correlation length; Universal amplitude ratios

E-mail: engels, fromme, seniuch@physik.uni-bielefeld.de 


\section{Introduction}

The Ising model is the simplest non-trivial spin model with critical behaviour. In three dimensions the universality class of the Ising model includes as well many real physical systems as also theoretical models which exhibit a continuous phase transition. Important examples are known from condensed matter physics and high-energy physics (see for instance the detailed review in Ref. [1]). Due to their simplicity and the many physically relevant applications the $3 d$ Ising model and members of the corresponding universality class belong to the most extensively studied systems. In these investigations both a variety of analytic methods [1]- 3] and Monte Carlo simulations have been used to calculate the universal quantities characterizing the universality class, such as critical exponents and amplitude ratios.

In the vicinity of the critical point the behaviour of the order parameter, the magnetization, and the correlation length are described by asymptotic scaling functions. The functions depend on the temperature and on the magnetic field and are universal apart from metric factors which are specific for the model under consideration. They are asymptotic in so far as they are valid in the thermodynamic limit, that is for $V \rightarrow \infty$, and for $T \rightarrow T_{c}$, so that irrelevant scaling fields can be neglected. In order to be comparable to other model systems the scaling functions have to be normalized which amounts to fixing the metric factors. There exist numerous parametrizations (see e. g. Refs. [1] and [4-[7]) of the equation of state, the scaling function of the order parameter, and other scaling functions. Since a parametric form of a scaling function has to describe the critical behaviour of the respective observable in accord with the known critical exponents and amplitude ratios these are usually taken either as an input to fix the parameters or as a check. Further critical quantities may then eventually be calculated from the parametrizations.

Instead of relying only on the known critical parameters one can in principle directly determine the scaling functions from simulation data, taking at most the critical exponents as input. This has already been achieved in the case of the threedimensional $O(2)$ [8] and $O(4)$ vector models [9, 10] for the equation of state. In the $3 d$ Ising model class (the $O(1)$ vector model) no such attempt has been undertaken as far as we know. In this paper our main intention is to accomplish the same for that universality class and to compare the parametric representation of the equation of state as found from field theory [3]-[5] directly to simulation results. A further aim is to find numerically the scaling function of the correlation length. It is clear, that for this project primarily simulations with non-zero magnetic field are required. The usual simulations at zero magnetic field can at best serve to normalize the scaling functions. The principal difficulties which we expect are due to the use of finite volumes in the simulations and to the finiteness of the critical region where scaling works. In order to attenuate these problems we use relatively large lattices with up to 120 points in each direction and we employ an improved Ising model to reduce corrections to scaling. 
The model which we want to study is the one-component $\phi^{4}$ or Landau-Ginzburg model. It is defined by

$$
\beta \mathcal{H}=-J \sum_{<x, y>} \phi_{x} \phi_{y}+\sum_{x}\left[\phi_{x}^{2}+\lambda\left(\phi_{x}^{2}-1\right)^{2}\right]-H \sum_{x} \phi_{x} .
$$

Here $x$ and $y$ are the nearest-neighbour sites on a three-dimensional simple cubic lattice, $\phi_{x}$ is the field variable at site $x$ and $H$ is the external magnetic field. We consider the coupling constant $J$ as inverse temperature, that is $J=1 / T$. In contrast to the ordinary Ising model the length of the spins $\phi_{x}$ in Eq. (1I) is not fixed to one. For $\lambda=0$ one obtains the Gaussian model, whereas in the limit $\lambda \rightarrow \infty$ the simple Ising model is recovered. By choosing an appropriate $\lambda$-value it is then possible to eliminate leading order corrections to scaling. This has been shown numerically in Refs. [1]-13] for $H=0$. There is however a drawback in using a finite $\lambda$ : we cannot directly verify our results by comparison with the known, non-universal amplitudes (see e. g. Ref. [14]) of the normal Ising model.

The observables which we want to measure in our simulations are the magnetization $M$, the susceptibility $\chi$ and the correlation length $\xi$. The magnetization is the expectation value of the lattice average $\phi$ of the spin variable

$$
M=\left\langle\frac{1}{V} \sum_{x} \phi_{x}\right\rangle=\langle\phi\rangle,
$$

where $V=L^{3}$ and $L$ is the number of lattice points per direction. As long as $H$ is non-zero and the volume large enough there is no problem with this definition. At zero magnetic field, $H=0$, however, the lattice average of the spins will have a vanishing expectation value on all finite lattices, that is $\langle\phi\rangle=0$. At very small magnetic fields and/or small $L$ we approximate $M$ therefore by [15]

$$
M \simeq\langle|\phi|\rangle \text {. }
$$

On finite lattices the magnetization of Eq. (3) approaches the infinite volume limit from above, whereas $M$ as defined by Eq. (2) for $H \neq 0$ reaches the thermodynamic limit from below. The susceptibility is defined as usual by the derivative of the magnetization

$$
\chi=\frac{\partial M}{\partial H}=V\left(\left\langle\phi^{2}\right\rangle-M^{2}\right) .
$$

For the determination of the correlation length we consider three different definitions. The second moment correlation length is obtained from

$$
\xi_{2 n d}=\left(\frac{\mu_{2}}{2 d \mu_{0}}\right)^{1 / 2},
$$

where the $\mu_{n}$ are the moments of the connected part of the correlation function

$$
\begin{aligned}
\mu_{n} & =\sum_{x} x^{n}\left\langle\phi_{x} \phi_{0}\right\rangle_{c}, \\
\left\langle\phi_{x} \phi_{0}\right\rangle_{c} & =\left\langle\phi_{x} \phi_{0}\right\rangle-\langle\phi\rangle^{2},
\end{aligned}
$$


and $x^{2}=\mathbf{x}^{2}$. The moment $\mu_{0}$ is equal to the susceptibility. A more and more often used definition of the correlation length is

$$
\xi^{F}=\left(\frac{\chi / F-1}{4 \sin ^{2}(\pi / L)}\right)^{1 / 2}
$$

where $F$ is the Fourier transform of the correlation function at momentum $p_{\mu}=$ $2 \pi \hat{e}_{\mu} / L$, and $\hat{e}_{\mu}$ a unit vector in one of the three directions

$$
F=\frac{1}{V}\left\langle\left|\sum_{x} \exp \left(i p_{\mu} x\right) \phi_{x}\right|^{2}\right\rangle .
$$

In the simulations we compute $F$ from an average over all three directions. Finally, the exponential or "true" correlation length $\xi_{\exp }$ governs the large distance behaviour of the connected part of the correlation function

$$
\left\langle\phi_{x} \phi_{0}\right\rangle_{c} \sim \exp \left(-|\mathbf{x}| / \xi_{\exp }\right) .
$$

We explain later in detail how we determine this observable from the correlation function data.

The rest of the paper is organized as follows. First we discuss the critical behaviour of the observables and the universal scaling functions, which we want to calculate. In Section 3 we describe some details of our simulations. Section 4 serves to determine the amplitudes of the magnetization and the susceptibility. In the following Section 5 we compare our data for the equation of state to a parametrization from field theory. The determination of the correlation length and its amplitudes is described in Section 6. In Section 7 we present our results for the scaling function of the correlation length. We close with a summary and the conclusions.

\section{Critical Behaviour and Scaling Functions}

In the thermodynamic limit $(V \rightarrow \infty)$ the observables show power law behaviour close to $T_{c}$. It is described by critical amplitudes and exponents of the reduced temperature $t=\left(T-T_{c}\right) / T_{c}$. The scaling laws at $H=0$ are for:

the magnetization

$$
M=B(-t)^{\beta} \quad \text { for } t<0
$$

the susceptibility

$$
\chi=C^{ \pm}|t|^{-\gamma} \text { for } t \rightarrow \pm 0,
$$

and the correlation length (without fixing for the moment a special definition)

$$
\xi=\xi^{ \pm}|t|^{-\nu} \quad \text { for } t \rightarrow \pm 0
$$


On the critical line $T=T_{c}$ or $t=0$ we have for $H>0$ the scaling laws

$$
\begin{gathered}
M=B^{c} H^{1 / \delta} \quad \text { or } \quad H=D_{c} M^{\delta}, \\
\chi=C^{c} H^{1 / \delta-1} \quad \text { with } \quad C^{c}=B^{c} / \delta,
\end{gathered}
$$

and for the correlation length

$$
\xi=\xi^{c} H^{-\nu_{c}}, \quad \nu_{c}=\nu / \beta \delta .
$$

We assume the following hyperscaling relations among the critical exponents to be valid

$$
2-\alpha=d \nu, \quad \gamma=\beta(\delta-1), \quad d \nu=\beta(1+\delta) .
$$

As a consequence only two critical exponents are independent. Because of the hyperscaling relations and the already implicitly assumed equality of the critical exponents above and below $T_{c}$ one can construct a multitude of universal amplitude ratios [16] (see also the discussion in Ref. [1]). The following list of ratios contains those which we will determine here

$$
\begin{aligned}
U_{2} & =C^{+} / C^{-}, & U_{\xi} & =\xi^{+} / \xi^{-}, \\
R_{\chi} & =C^{+} D_{c} B^{\delta-1}, & Q_{c} & =B^{2}\left(\xi^{+}\right)^{d} / C^{+}
\end{aligned}
$$

and

$$
Q_{2}=\left(\xi^{c} / \xi^{+}\right)^{\gamma / \nu} C^{+} / C^{c}
$$

The critical behaviours of the magnetization and the susceptibility originate from the singular part of the free energy density. In the thermodynamic limit it obeys the scaling law

$$
f_{s}\left(u_{t}, u_{h}\right)=b^{-d} f_{s}\left(b^{y_{t}} u_{t}, b^{y_{h}} u_{h}\right) .
$$

Here $b$ is a free length rescaling factor, and

$$
y_{t}=1 / \nu, \quad y_{h}=1 / \nu_{c}
$$

We have neglected possible dependencies on irrelevant scaling fields $u_{i}$ with exponents $y_{i}<0$. Close to $T_{c}$ and for $H \rightarrow 0$ the remaining relevant scaling fields $u_{t}$ and $u_{h}$ are proportional to the reduced temperature, $u_{t} \sim t$, and the magnetic field, $u_{h} \sim H$, that is one can replace $u_{t}$ and $u_{h}$ by a normalized reduced temperature $\bar{t}=t T_{c} / T_{0}$ and magnetic field $h=H / H_{0}$. To be definite we assume in the following that $H$ is positive. Choosing the scale factor $b$ such that $b^{y_{h}} h=1$ we find

$$
f_{s}=h^{d \nu_{c}} \Phi_{s}\left(\bar{t} / h^{1 / \beta \delta}\right)
$$

and using $M=-\partial f_{s} / \partial H$ we arrive at

$$
M=h^{1 / \delta} f_{G}\left(\bar{t} / h^{1 / \beta \delta}\right),
$$


where $\Phi_{s}$ and $f_{G}$ are universal scaling functions once the normalizations are fixed. The relation (24) is one form of the magnetic equation of state. Alternatively, one may use the Widom-Griffiths form of the equation of state [17, 18]

$$
y=f(x),
$$

where

$$
y \equiv h / M^{\delta}, \quad x \equiv \bar{t} / M^{1 / \beta} .
$$

We take the standard normalization conditions

$$
f(0)=1, \quad f(-1)=0
$$

which imply

$$
\begin{gathered}
M(t=0)=h^{1 / \delta} \quad \text { and } \quad H_{0}=D_{c}, \\
M(h=0)=(-\bar{t})^{\beta} \quad \text { and } \quad T_{0}=B^{-1 / \beta} T_{c} .
\end{gathered}
$$

The two forms (24) and (25) are of course equivalent. The function $f_{G}(z)$ and its argument $z$ are related to $x$ and $y$ by

$$
f_{G}=y^{-1 / \delta}, \quad z \equiv \bar{t} / h^{1 / \beta \delta}=x y^{-1 / \beta \delta} .
$$

Correspondingly the normalization conditions (27) translate into

$$
f_{G}(0)=1, \text { and } f_{G}(z) \underset{z \rightarrow-\infty}{\longrightarrow}(-z)^{\beta} .
$$

Since the susceptibility $\chi$ is the derivative of $M$ with respect to $H$ we obtain from Eq. (24)

$$
\chi=\frac{\partial M}{\partial H}=\frac{h^{1 / \delta-1}}{H_{0}} f_{\chi}(z)
$$

with

$$
f_{\chi}(z)=\frac{1}{\delta}\left(f_{G}(z)-\frac{z}{\beta} f_{G}^{\prime}(z)\right) .
$$

The asymptotic behaviour of $f_{\chi}$ for $H \rightarrow 0$ at fixed $t$, that is for $z \rightarrow \pm \infty$, is determined by Eq. (12)

$$
f_{\chi}(z) \underset{z \rightarrow \pm \infty}{=} C^{ \pm} D_{c} B^{\delta-1}( \pm z)^{-\gamma} .
$$

We note, that the amplitude for $z \rightarrow \infty$ is simply the universal ratio $R_{\chi}$, whereas for $z \rightarrow-\infty$ it is the analogous quantity $R_{\chi}^{-}=R_{\chi} / U_{2}$. The leading terms for $f_{G}$ are respectively

$$
\begin{aligned}
& f_{G}(z) \underset{\substack{z \rightarrow-\infty \\
=}}{=}(-z)^{\beta}+R_{\chi}^{-}(-z)^{-\gamma}, \\
& f_{G}(z) R_{\chi} z^{-\gamma},
\end{aligned}
$$

in accord with the normalization (31). The asymptotical identity $f_{G}=f_{\chi}$ for $z \rightarrow \infty$ is due to the fact that for $T>T_{c}$ and small magnetic field $M$ is proportional to $H$. 
The correlation length fulfills a scaling law analogous to the one of the free energy density

$$
\xi\left(u_{t}, u_{h}\right)=b \xi\left(b^{y_{t}} u_{t}, b^{y_{h}} u_{h}\right) .
$$

In the same way as before we obtain from this equation the dependence of the correlation length on $\bar{t}$ and $h$ in the critical region and the thermodynamic limit

$$
\xi=h^{-\nu_{c}} g_{\xi}(z),
$$

in terms of a scaling function $g_{\xi}(z)$. Here, we have assumed that the metric factors in the scaling functions for $f_{s}$ and $\xi$ are the same, which is the usual assumption [16. The function $g_{\xi}(z)$ is then universal except for one normalization factor. On the critical line $t=0$ or $z=0$ we find from (16)

$$
g_{\xi}(0)=\xi^{c} D_{c}^{-\nu_{c}}=\xi^{c}\left(B^{c}\right)^{\nu / \beta}
$$

and from (13) the asymptotic behaviour at $z \rightarrow \pm \infty$

$$
g_{\xi}(z) \underset{z \rightarrow \pm \infty}{=} \xi^{ \pm} B^{\nu / \beta}( \pm z)^{-\nu}
$$

Indeed, the ratio of the amplitude for $z \rightarrow \infty$ in (40) and $g_{\xi}(0)$ is universal

$$
\frac{\xi^{+} B^{\nu / \beta}}{\xi^{c}\left(B^{c}\right)^{\nu / \beta}}=\left(\frac{\delta R_{\chi}}{Q_{2}}\right)^{\nu / \gamma},
$$

whereas $g_{\xi}(0)$ itself is not universal. Pelissetto and Vicari [1] associate a universal scaling function $f_{\xi}(x)$ to the correlation length by the relation

$$
\xi^{2}=\left(B^{c}\right)^{2 \delta / d} M^{-2 \nu / \beta} f_{\xi}(x),
$$

where $x$ is defined as in (26). It is straightforward to show that

$$
\frac{f_{\xi}(x)}{f_{\xi}(0)}=\left(\frac{g_{\xi}(z)}{g_{\xi}(0)} \cdot f_{G}(z)^{\nu / \beta}\right)^{2} .
$$

The value $x=0$ corresponds to $z=0$. Since both $f_{\xi}(x)$ and $f_{G}(z)$ are universal this is also true for

$$
\hat{g}_{\xi}(z)=g_{\xi}(z) / g_{\xi}(0) .
$$

As we shall see, the scaling functions of the correlation length and of the susceptibility have similar shapes, each with a peak at a positive $z$-value. It is therefore useful to consider the ratio $\xi^{2} / \chi$. We can express it with the corresponding ratio of universal scaling functions

$$
\frac{\xi^{2}}{\chi}=\left(\xi^{c}\right)^{2}\left(B^{c}\right)^{-1} H^{-\eta \nu_{c}} \cdot \frac{\hat{g}_{\xi}^{2}(z)}{f_{\chi}(z)} .
$$

Here $\eta=2-\gamma / \nu$ is the exponent describing the behaviour of the two-point function at the critical point. We postpone the discussion of parametrizations of the scaling functions to the sections where we compare them to our data. 


\section{Simulation Details}

In the numerical simulation of the model (1) we followed the examples given by Brower and Tamayo [19] and Hasenbusch [13. They combined a simple local Metropolis algorithm for the update of the field with a cluster algorithm, which only updates the sign of the fields $\phi_{x}$ at fixed values of the modulus $\left|\phi_{x}\right|$. During the cluster update the system is therefore treated as an Ising model with link-dependent coupling constants $J_{x y}=J\left|\phi_{x}\right|\left|\phi_{y}\right|$ and local magnetic fields $H_{x}=H\left|\phi_{x}\right|$. In order to achieve ergodicity of the entire update process the two algorithms have to be used in an alternating manner. In a local Metropolis step a new proposal $\phi_{x}^{\prime}$ for the field is produced by [13]

$$
\phi_{x}^{\prime}=\phi_{x}+s\left(r-\frac{1}{2}\right)
$$

where $r$ is a uniformly in $(0,1]$ distributed random number and $s$ an adjustable size parameter. The prescription (46) obviously allows for changes of the lengths of the spins. For the cluster algorithm we chose Wolff's single cluster update [20], which was modified to include a non-zero magnetic field [21]. This variant was also successfully used in previous simulations of the $O(2)$ [ and $O(4)$ spin models [10]. A complete Monte Carlo update consists then of $n$ consecutive cluster updates followed by a Metropolis sweep of the entire lattice.

As explained in the introduction we aim at minimizing the corrections to scaling. We have therefore fixed the parameter $\lambda$ of the model to the optimal value $\lambda=1.1$ found by Hasenbusch [13]. The very precise value $J_{c}=0.3750966(4)$ for the corresponding critical coupling was adopted from the same paper. All our simulations were done on three-dimensional lattices with periodic boundary conditions and linear extensions ranging from $L=24$ to $L=120$. The size parameter $s$ in Eq. (46) was set to $s=2$. That resulted in acceptance rates of $59 \%$ to $67 \%$ in the Metropolis step for the simulated $J$ and $H$-values. The number $n$ of cluster updates was chosen in the region $10 \leq n \leq 25$ for $T>T_{c}$ and in the cold phase $\left(T<T_{c}\right)$ in the range $3 \leq n \leq 8$. Between two measurements of the observables 20 to 50 Monte Carlo updates were performed, such that the integrated autocorrelation times of the energy and the magnetization were $\tau_{\text {int }} \lesssim 3$ for $L \leq 48$ and $\tau_{\text {int }} \lesssim 5$ for $L \geq 72$. In general we made 20000 measurements, for $L=24$ sometimes 40000 .

The coupling constant region which we have explored was $0.365 \leq J \leq 0.4$, the magnetic field was varied from $H=0$ to $H=0.005$. The critical region, where asymptotic scaling works, is clearly inside this parameter range. We come back to this point when we discuss our data for the scaling functions. Further details on the Monte Carlo update, for example on the distribution of the modulus of the spins, can be found in Ref. [22]. 


\section{The Critical Amplitudes of $M$ and $\chi$}

We consider first our results for the magnetization at $H=0$ and $T<T_{c}$. The primary objective here is to determine the normalization $T_{0}$ or equivalently the critical amplitude $B$ of (11). In Fig. 11 we show $M$ from Eq. (3) as a function of $T_{c}-T$ for different lattice sizes. The temperatures correspond to coupling constants in the range $0.376 \leq J \leq 0.4$. In order to eliminate finite-size effects at a fixed $J$ (or $T$ ), we have simulated at increasingly larger values of $L$.

We have fitted the results from the largest lattices to the following ansatz taking into account possible analytic and non-analytic corrections to scaling

$$
M=b_{0}\left(T_{c}-T\right)^{\beta}\left[1+b_{1}\left(T_{c}-T\right)^{\omega \nu}+b_{2}\left(T_{c}-T\right)\right] .
$$

For the fits we used the critical exponents of Ref. 3] as input

$$
\beta=0.3258(14), \quad \nu=0.6304(13), \quad \omega=0.799(11)
$$

because we wanted to test the corresponding equation of state. The remaining exponents were determined from the hyperscaling relations (17). Instead of these field theory results we could as well have used the exponents of Hasenbusch [13] or the estimates proposed by Pelissetto and Vicari [1]. The difference is marginal, the various exponent results coincide within the error bars. The fit with the ansatz (47) nicely confirms the expected absence of the leading non-analytic correction term: $b_{1}$ is zero within error bars. The best fit to the points with $T_{c}-T \lesssim 0.10$ and $b_{1} \equiv 0$

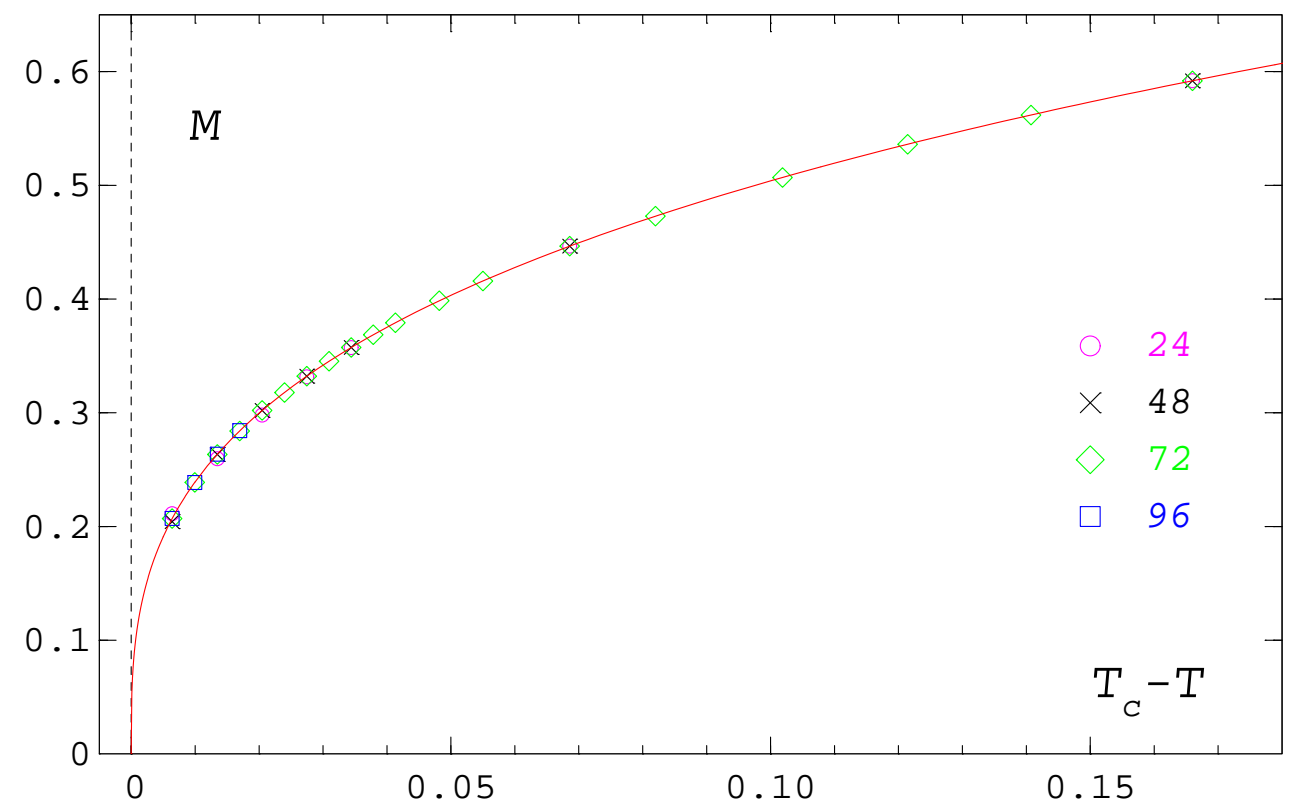

Figure 1: The magnetization $M$ at $H=0$ versus $T_{c}-T$ for $L=24,48,72$ and 96 . The solid line shows the fit (49). 


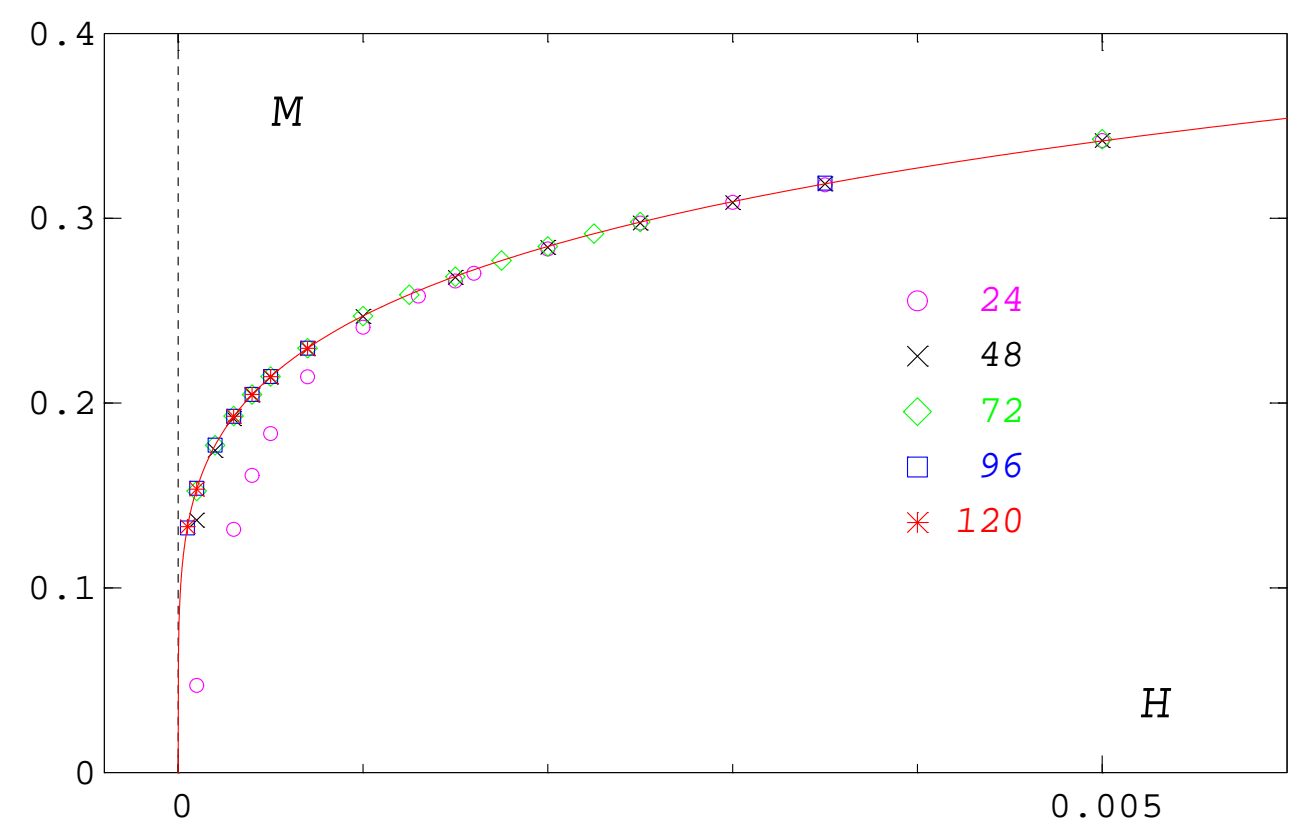

Figure 2: The magnetization $M$ at $T_{c}$ as a function of the magnetic field $H$ for $L=24,48,72,96$ and 120. The solid line shows the fit (53).

is given by

$$
M=1.0735(2)\left(T_{c}-T\right)^{\beta}\left[1-0.061(2)\left(T_{c}-T\right)\right],
$$

with $\chi^{2} / N_{f}=0.8$. From $b_{0}$ we obtain

$$
T_{0}=b_{0}^{-1 / \beta}=0.8044(4), \quad B=b_{0} T_{c}^{\beta}=1.4776(2) .
$$

On the critical isotherm, that is at $T_{c}$, we have measured the magnetization for $H>0$ on lattices with $L=24,48,72,96$ and 120 using Eq. (2). The results are shown in Fig. 2. We observe an increasing finite size dependence close to $H=0$. The results from the largest lattices have been fitted to the ansatz

$$
M=B^{c} H^{1 / \delta}\left[1+B_{1}^{c} H^{\omega \nu_{c}}+B_{2}^{c} H\right] .
$$

In the $H$-interval $[0.00005,0.0005]$, however, only the leading term is of relevance. A fit in this interval with simply the first term in (51) leads to

$$
B^{c}=1.0435(15) \text {. }
$$

The remaining amplitudes $B_{1,2}^{c}$ may then be determined from a subsequent fit to all available data. As on the coexistence line we find that the value for the leading non-analytic correction amplitude $B_{1}^{c}$ is small and of the same size as its error. We have therefore made fits with fixed $B_{1}^{c} \equiv 0$ in the region $0.00005 \leq H \leq 0.0025$ and find

$$
M=1.0435(15) H^{1 / \delta}[1-2.65(5) H]
$$



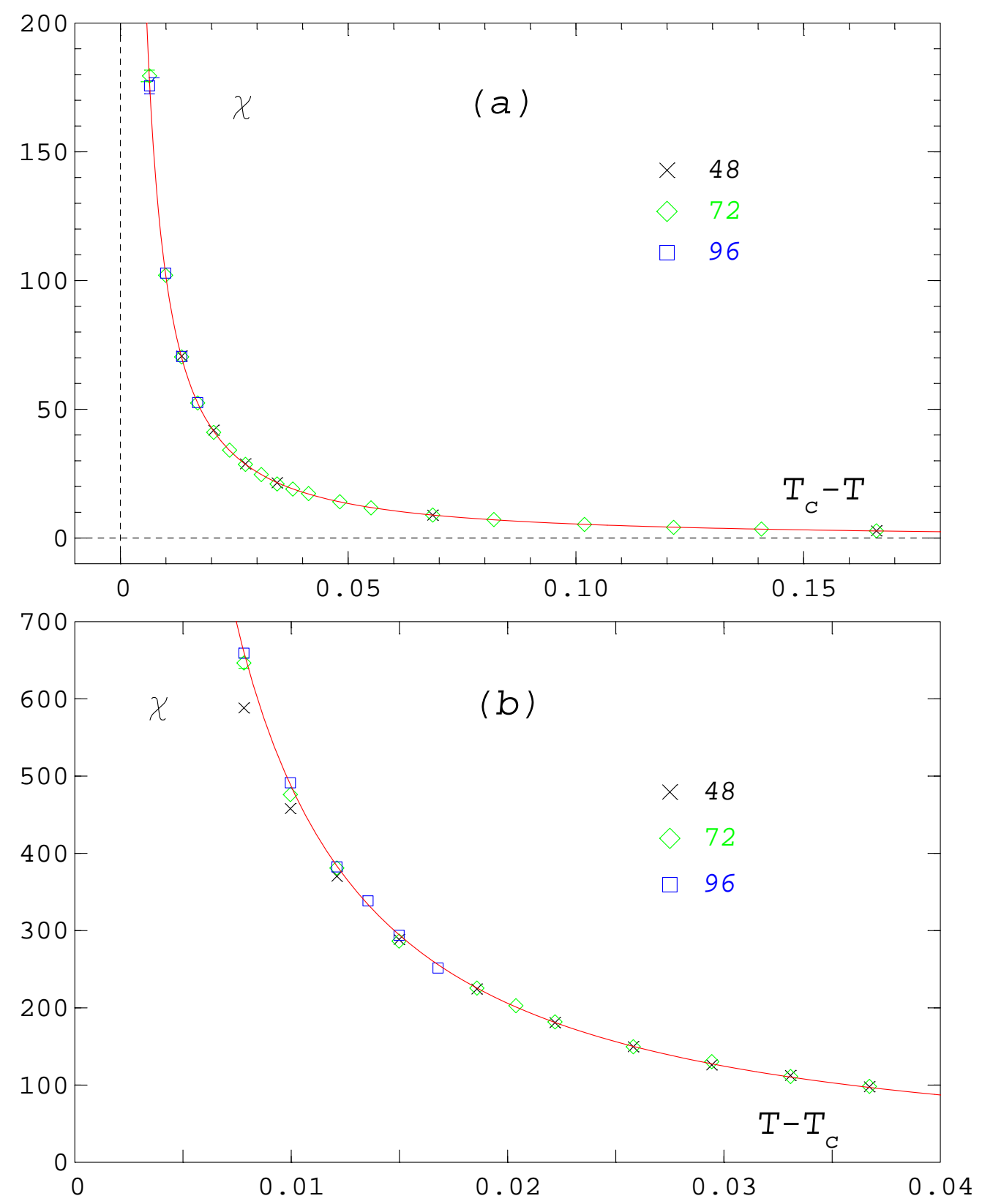

Figure 3: The susceptibility $\chi$ at $H=0$ for $L=48,72$ and 96, (a) below $T_{c}$ versus $T_{c}-T$ and (b) above $T_{c}$ versus $T-T_{c}$. The solid lines show the fits from Eqs. (55) and (56), respectively.

with $\chi^{2} / N_{f} \approx 2$. From the value of $B^{c}$ in (52) we can immediately deduce the values of two further quantities

$$
C^{c}=0.2172(3) \quad \text { and } \quad D_{c}=H_{0}=0.8150(56)
$$

Our next aim is the determination of the critical amplitudes $C^{ \pm}$. To this end we have evaluated the data of the susceptibility below and above the critical temperature for $H=0$. The data points are plotted in Figs. 3 (a) and (b) as a function of 
$\left(T_{c}-T\right)$ and $\left(T-T_{c}\right)$, respectively. Inspired by our experience with the magnetization in the cold phase, we have directly used a fit ansatz without a non-analytic correction term. The data points from the largest lattices in the range $0.01 \leq T_{c}-T \lesssim 0.10$ lead to

$$
\chi=0.1006(5)(-t)^{-\gamma}[1-2.0(3)(-t)],
$$

with $\chi^{2} / N_{f} \approx 1.3$. Note that here we have used the reduced temperature as variable. A corresponding ansatz in the symmetric phase $\left(T>T_{c}\right)$ shows that the correction term is zero inside the error bars. The points from the largest lattices are however well described by the leading term only with

$$
\chi=0.4785(15) t^{-\gamma}
$$

Here, $\chi^{2} / N_{f} \approx 1.2$. From the fits (55) and (156) we have

$$
C^{-}=0.1006(5) \text { and } C^{+}=0.4785(15) \text {. }
$$

This enables us to calculate the two universal amplitude ratios

$$
U_{2}=4.756(28) \text { and } R_{\chi}=1.723(13)
$$

The ratio $U_{2}$ is in excellent agreement with two other MC results: 4.75(3) from the standard Ising model [23] and 4.72(11) from (3+1)-dimensional $S U(2)$ gauge theory 24, as well as other results (see Table 11 in [1] and Table 5 in [3]). The ratio is also well inside the bounds [4.688,4.891] derived in Ref. [25]. The result for $R_{\chi}$ is the first one solely from MC calculations. It is in agreement with 1.57(23) from Refs. [14, 26] and slightly higher than the result 1.660(4) from Refs. [7, 27].

\section{The Magnetic Equation of State}

After having determined the normalizations $T_{0}$ and $H_{0}$ we can calculate the universal scaling function of the magnetization for the Ising class. To this end we have performed further simulations at 14 couplings in the range $0.385 \leq J \leq 0.365$ with external fields $H$ varying between 0.0001 and 0.005 . We convinced ourselves that the used lattice extensions were large enough to produce volume independent results. For that purpose $L=48$ was sufficient at high temperatures (small $J$ ), closer to $T_{c}$ lattices with linear extension $L=72$ and 96 were needed. The finite size effects diminished with increasing field, generally they were stronger in the cold phase than in the hot phase. In Fig. 4 we present the results for $f_{G}(z)$ obtained from the magnetization data in the coupling range $0.38 \leq J \leq 0.37$. Obviously these data are scaling well. Since the data from the couplings $J=0.385$ and 0.365 showed already slight deviations from scaling behaviour we have discarded them. Similarly, limitations were found for the scaling $H$-regions. The data for $f_{G}(z)$ were scaling for $J=0.38$ only up to $H=0.0003$, for $J=0.378$ to $J=0.375$ up to $H=0.0005$ and 


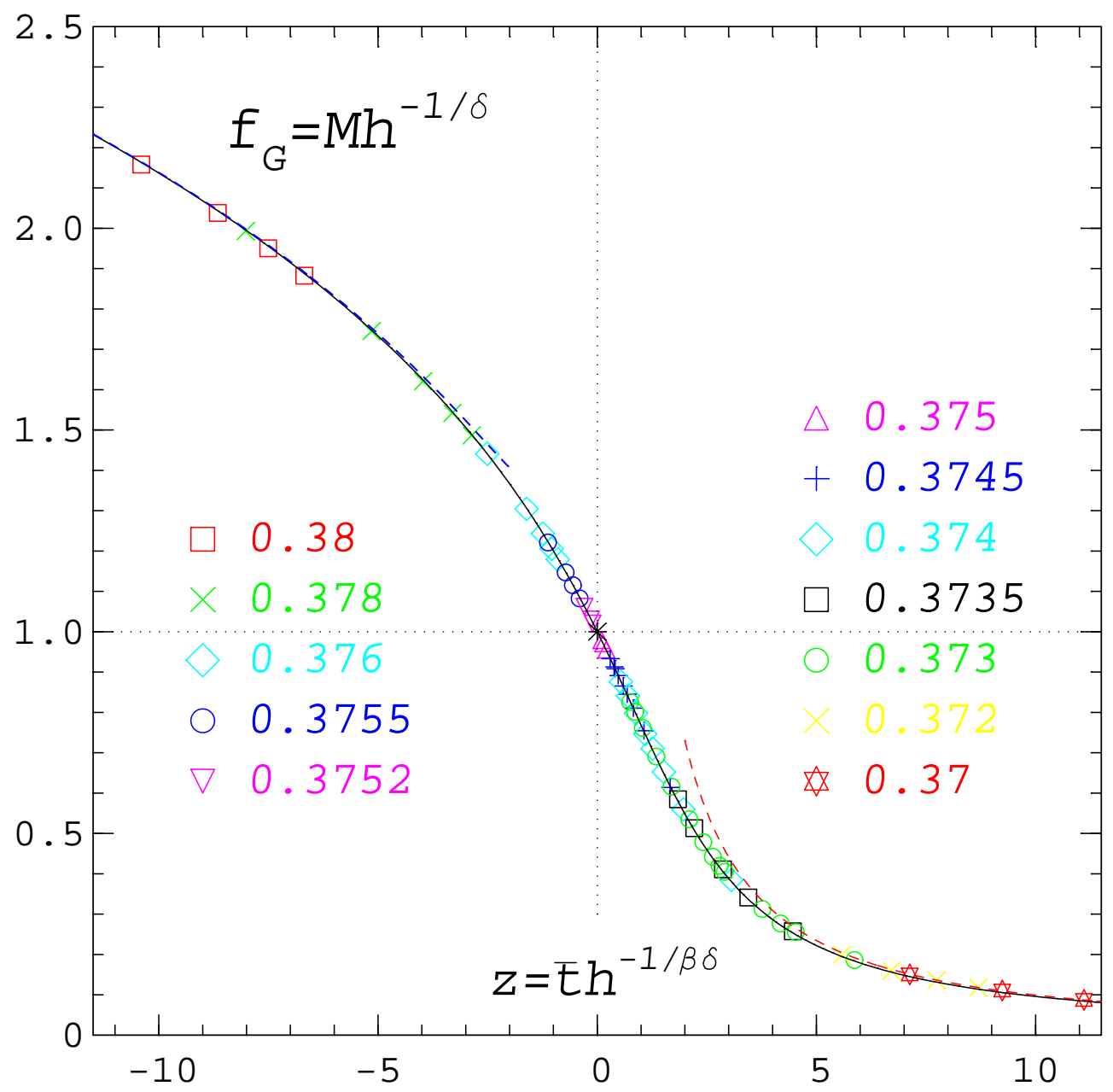

Figure 4: The equation of state: $f_{G}=M h^{-1 / \delta}$ as a function of $z=\bar{t} h^{-1 / \beta \delta}$. The solid line shows the parametrization of Ref. [3], the dashed lines the asymptotic forms (35) and (36). The numbers refer to the different $J=1 / T$-values of the data, the dotted lines cross at the normalization point $f_{G}(0)=1$.

for $J=0.3745$ to $J=0.372$ up to $H=0.0015$. At $J=0.37$ the scaling $H$-range was limited again by $H=0.0003$. Only data within these parameter ranges are shown in Fig. 4. From the amplitude ratios $U_{2}$ and $R_{\chi}$ in (58) one can calculate the asymptotic forms (35) and (36) of $f_{G}(z)$. As can be seen in Fig. 4, the data for $f_{G}$ coincide with the corresponding asymptotic form already for $z \lesssim-4$ and $z \gtrsim 6-7$.

The numerically determined scaling function can now be compared to parametric representations of the equation of state. A prominent and widely accepted representation of the equation of state is that of Guida and Zinn-Justin [3]- [5], which is based on field theory methods. They parametrize the equation of state in terms of two new variables $R$ and $\theta$ by setting [28]-30]

$$
\begin{aligned}
M & =m_{0} R^{\beta} \theta, \\
\bar{t} & =R\left(1-\theta^{2}\right),
\end{aligned}
$$




$$
h=h_{0} R^{\beta \delta} \hat{h}(\theta)
$$

where $m_{0}$ and $h_{0}$ are two normalization constants. Because $M$ is linear in $\theta$ this is also known as the linear model. The function $\hat{h}(\theta)$ is an odd function and was approximated by a 5 th order polynomial with the following coefficients [3]

$$
\hat{h}(\theta)=\theta-0.76201(36) \theta^{3}+0.00804(11) \theta^{5} .
$$

In Refs. 4, 5, slightly different coefficients are given. The function $\hat{h}(\theta)$ in (62) has two zeros. The one at $\theta=0$ corresponds to $h=0, t>0$, the second at $\theta_{0}=1.154$ to the coexistence line $h=0, t<0$. For $\theta=1$ one obtains the critical line $t=0$. Using the normalization conditions (27) one finds

$$
m_{0}=\frac{\left(\theta_{0}^{2}-1\right)^{\beta}}{\theta_{0}}, \quad h_{0}=\frac{m_{0}^{\delta}}{\hat{h}(1)} .
$$

The Widom-Griffiths scaling variables $x$ and $y$ are only functions of $\theta$

$$
x=\frac{1-\theta^{2}}{\theta_{0}^{2}-1}\left(\frac{\theta_{0}}{\theta}\right)^{1 / \beta}, \quad y=\frac{\hat{h}(\theta)}{\hat{h}(1) \theta^{\delta}} .
$$

The same applies to the scaling function $f_{G}$ and its argument $z$

$$
z=\frac{1-\theta^{2}}{\theta_{0}^{2}-1} \theta_{0}^{1 / \beta}\left(\frac{\hat{h}(\theta)}{\hat{h}(1)}\right)^{-1 / \beta \delta}, \quad f_{G}=\theta\left(\frac{\hat{h}(\theta)}{\hat{h}(1)}\right)^{-1 / \delta} .
$$

In Fig. 4 the solid line represents the parametrization from Eq. (65) with $\hat{h}(\theta)$ from (62). Evidently, the agreement between the data and the solid line is excellent. Also, the asymptotic form for $z<0$ coincides directly with the parametrization in a large range, whereas the asymptotic form for $z>0$ is marginally higher in the shown large $z$-range.

We have also plotted the data in the Widom-Griffiths form. The small $x$-region is shown in Fig. 5 (a), the large $x$-region in Fig. 5 (b). This kind of representation of the equation of state is asymmetric with respect to the critical point: the cold or broken phase $\left(T<T_{c}\right)$ extends in $x$ from -1 to 0 and in $y$ from 0 to 1 , the hot phase $\left(T>T_{c}\right)$ reaches in $x$ from 0 to infinity and in $y$ from 1 to infinity. Since the magnetization appears here in both variables small deviations from scaling are easier to detect than in Fig. 4. This applies in particular to the cold phase. Therefore we included only points for $x<0$ in Fig. 5 (a) with $H \leq 0.0003$, because the points with larger $H$-values exhibited already very slight, but perceptible deviations. In the hot phase we could use all the points already displayed in Fig. 4. As in Fig. 4, the data show in the Widom-Griffiths representation a similarly impressive agreement with the parametrization of the equation of state by Guida and Zinn-Justin.

The scaling function $f_{\chi}(z)$ of the susceptibility is connected via Eq. (33) to the scaling function $f_{G}(z)$ of the magnetization. It is therefore in principal known. On 

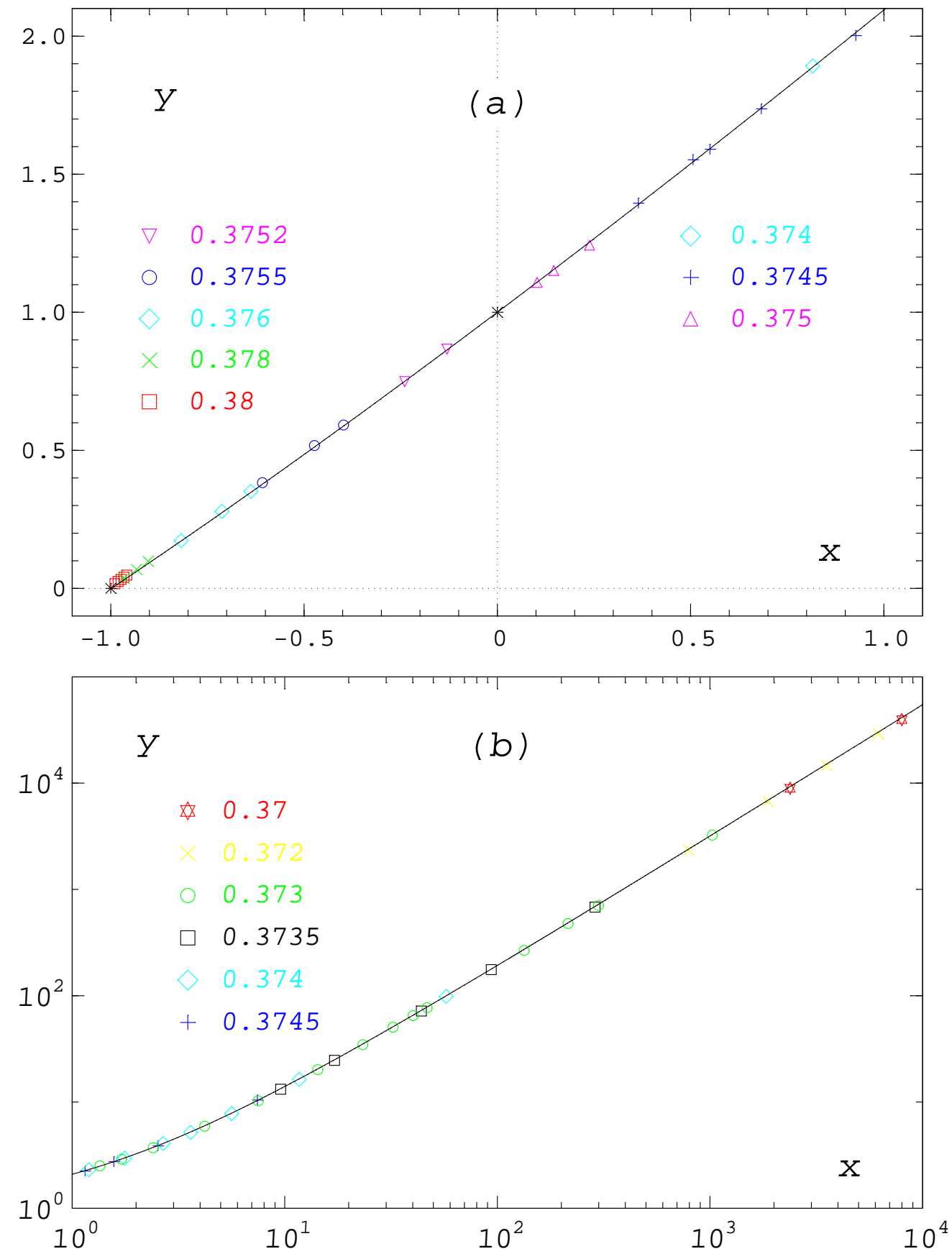

Figure 5: The Widom-Griffiths form of the equation of state: $y=h / M^{\delta}$ as a function of $x=\bar{t} / M^{1 / \beta}$. The solid lines show the parametrization of Ref. [3], the numbers refer to the $J=1 / T$-values of the data. Part (a) of the figure displays the small $x$-region where the stars denote the normalizations (27), part (b) the large $x$-region.

the other hand we have direct data for $\chi$ from our simulations which allows for another check of parametric representations and the scaling hypothesis. In Fig. [6]

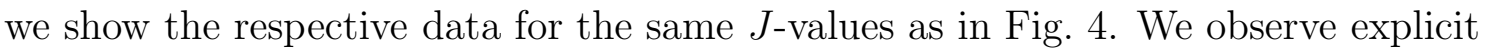




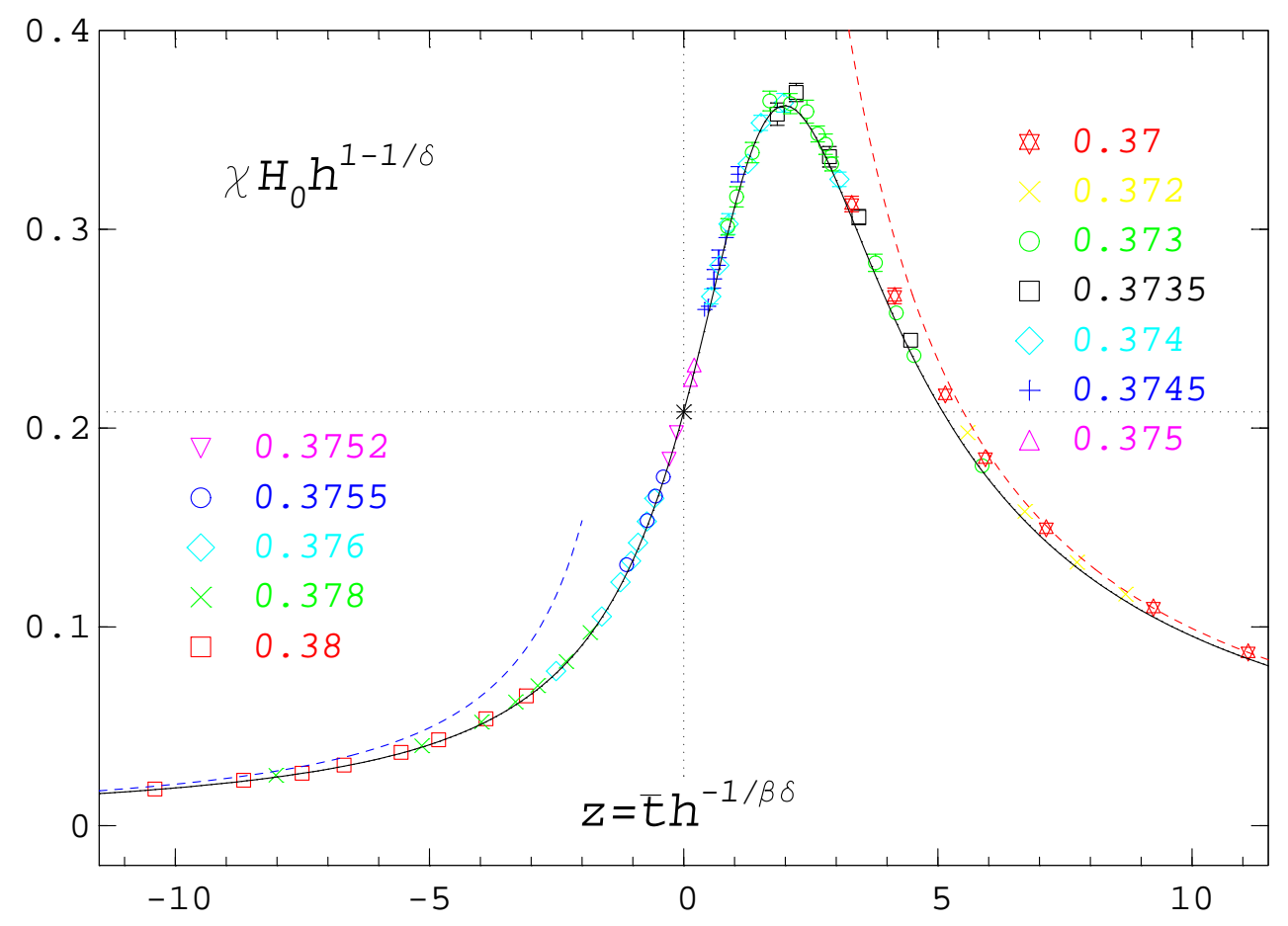

Figure 6: The scaling function of the susceptibility $f_{\chi}(z)=\chi H_{0} h^{1-1 / \delta}$. The solid line is the parametrization of Ref. [3], the dashed lines are the asymptotic forms (34). The numbers refer to the $J=1 / T$-values of the data. The star represents the normalization $f_{\chi}(0)=1 / \delta$.

scaling of the data, sometimes even in a larger $H$-range. In comparison to the parametrization of Ref. [3] we find complete agreement in the cold phase $(z<0)$ and a slightly increasing small difference to the data in the hot phase $(z>0)$. The reason for this little discrepancy lies in the different asymptotic amplitudes which are used in the parametrization and which we measured from our data. The amplitude in question is $R_{\chi}$. In the parametrization scheme one derives

$$
R_{\chi}=\hat{h}(1) \theta_{0}^{\gamma / \beta}\left(\theta_{0}^{2}-1\right)^{-\gamma}
$$

This results in the number $R_{\chi}=1.666$, whereas we found from our amplitude measurements on the critical line and at $h=0$ the value $R_{\chi}=1.723(13)$, that is a slightly higher number. The data shown in Fig. [6] are consistent with that finding: at larger $z$-values they coincide with the corresponding asymptotic curve.

\section{The Correlation Length}

Instead of using correlation functions of the individual spins it is more favourable 31] to consider spin averages over planes and their respective correlation functions. 
For example, the spin average over the $(x, y)$-plane at position $z$ is defined by

$$
S_{z}=\frac{1}{L^{2}} \sum_{x, y} \phi_{x, y, z} .
$$

The average $S$ of all $S_{z}$ is equal to the lattice average $\phi$ and

$$
\left\langle S_{z}\right\rangle=\langle S\rangle=\langle\phi\rangle
$$

Correspondingly, we define the plane-correlation function $G(z)$ by

$$
G(z)=L^{2}\left(\left\langle S_{0} S_{z}\right\rangle-\langle S\rangle^{2}\right)
$$

Here, $z$ is the distance between the two planes. Instead of choosing the $z$-direction as normal to the plane one can as well take the $x$ - or $y$-directions. Accordingly, we enhance the accuracy of the correlation function data by averaging over all three directions and all possible translations. The correlators are symmetric and periodic functions of the distance $\tau$ between the planes

$$
G(\tau)=G(-\tau), \quad \text { and } \quad G(\tau)=G(L-\tau) .
$$

The factor $L^{2}$ on the right-hand side of (69) ensures the relation

$$
\chi=\sum_{\tau=0}^{L-1} G(\tau)=\sum_{\tau=-L / 2+1}^{L / 2} G(\tau) .
$$

Similarly, the second moment $\mu_{2}$ may be expressed in terms of the plane correlators as

$$
\mu_{2}=d \sum_{\tau=-L / 2+1}^{L / 2} \tau^{2} G(\tau)
$$

Like the point-correlation functions in Eq. (10) the plane correlators $G(\tau)$ decay exponentially. In Fig. [7 we show the logarithm of typical plane-correlation functions for different $L$, here at $T=T_{c}$ and $H=0.0003$. The expected behaviour is actually confirmed, as well as the influence of periodic boundary conditions on finite lattices. In order to obtain the exponential correlation length from $G(\tau)$ on a lattice with linear extension $L$ and periodic boundary conditions we therefore make the ansatz

$$
G(\tau)=A[\exp (-\tau / \xi)+\exp (-(L-\tau) / \xi)],
$$

and then try to fit the data for $G(\tau)$ in an appropriate $\tau$-range. The ansatz (73) implies of course, that there are no additional excitations contributing to $G(\tau)$. Inspired by the experiences reported in [23], we proceed in the following way. First we calculate an effective correlation length $\xi_{\text {eff }}(\tau)$ from (73) using only the correlators at $\tau$ and $\tau+1$. For $\tau \ll L$ this correlation length is approximately given by

$$
\xi_{\text {eff }}(\tau)=\frac{-1}{\ln (G(\tau+1) / G(\tau))} .
$$




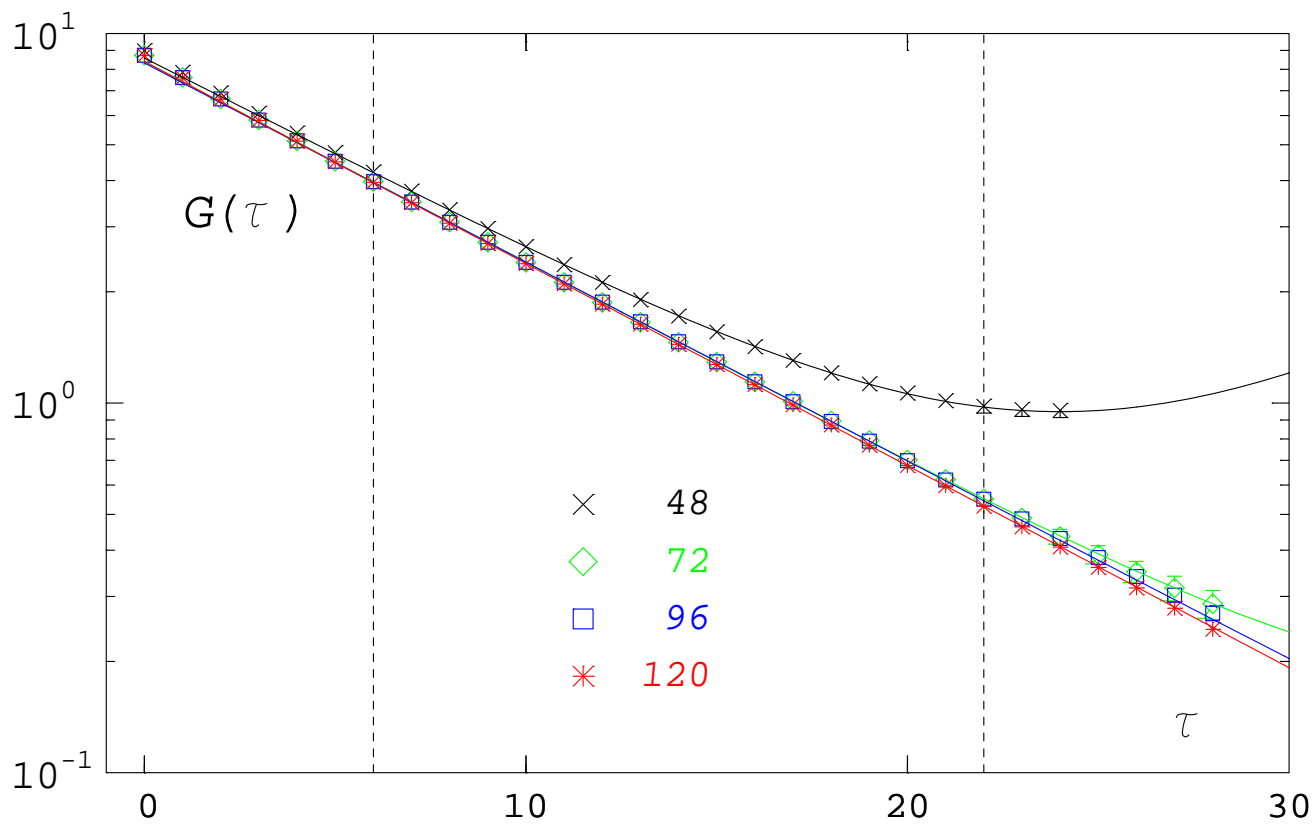

Figure 7: The plane-correlation function $G(\tau)$ at $T=T_{c}$ and $H=0.0003$ for $L=48,72,96$ and 120 . The solid lines show the fits from the ansatz (73), the dashed lines indicate the $\tau$-range, inside which the fits were done.

With increasing $\tau$ also $\xi_{\text {eff }}$ increases and eventually reaches a plateau inside its error bars. Most likely, the lower values of $\xi_{\text {eff }}$ at small $\tau$ are due to higher excitations. At large distances $\tau$ the resulting $\xi_{\text {eff }}$ start to fluctuate when the relative error of the data becomes too large. The two limits define an intermediate $\tau$-range where a global fit with the ansatz (73) can finally be used to estimate the exponential correlation length.

In order to give an impression of the actual implementation of this procedure we show in Table 1 the results of the fits $\left(\xi^{\text {fit }}\right)$ to the plane-correlation functions from Fig. [7. The corresponding correlation length is here close to 8. The plateau of $\xi_{\text {eff }}$ is reached for all $L$ at a value for $\tau_{\min }$ between 6 and 10. A reasonable upper fit limit is $\tau_{\max }=18$ to 22 . Varying $\tau_{\max }$ in this range does not affect the fit result. Also a variation of $\tau_{\text {min }}$ from 6 to 10 leads only to changes inside the error bars. The respective $\chi^{2} / N_{f}$ are less than or equal to 0.02 . If we compare $\xi^{f i t}$ to the effective correlation length at $\tau_{\min }$, we find essentially the same result, including the size of the error bar.

We have calculated as well the correlation length $\xi^{F}$ from Eq. (8). We see from Table 1, that $\xi^{F}$ is compatible with $\xi^{\text {fit }}$, but has a larger error. Only for the largest lattice with $L=120$ we obtain a difference. This may be due to the use of all correlations in the calculation of the Fourier transform, also those at large distances, where the relative errors are large. In addition, the numerical determination of $\xi^{F}$ requires an increasing accuracy with increasing $L$ because of the diminishing denomiator $\sin (\pi / L)$ in Eq. (8) and the difference $\chi / F-1$ in the nominator. 


\begin{tabular}{|r||r|c|l|c||c|c|}
\hline$L$ & $\tau_{\min }$ & $\tau_{\max }$ & $\xi^{\text {fit }}$ & $\xi_{\text {eff }}\left(\tau_{\min }\right)$ & $\xi^{F}$ & $\xi_{2 n d}$ \\
\hline 48 & 7 & 18 & $8.265(104)$ & $8.278(79)$ & $8.18(16)$ & $6.80(11)$ \\
72 & 6 & 20 & $7.986(45)$ & $7.917(50)$ & $7.92(15)$ & $7.52(17)$ \\
96 & 8 & 19 & $8.055(50)$ & $8.011(49)$ & $8.18(16)$ & $8.10(21)$ \\
120 & 10 & 22 & $7.957(44)$ & $7.948(49)$ & $7.43(16)$ & $7.51(10)$ \\
\hline
\end{tabular}

Table 1: The correlation length at $T=T_{c}$ and $H=0.0003$ from different methods: $\xi^{\text {fit }}$ from fits to the ansatz (73) in the $\tau$-interval $\left[\tau_{\min }, \tau_{\max }\right] ; \xi^{F}$ from Eq. (8) and $\xi_{2 n d}$ from Eq. (15) using Eq. (72). For comparison we show also $\xi_{\text {eff }}\left(\tau_{\min }\right)$.

The accurate numerical calculation of $\xi_{2 n d}$ is also problematic. The reason for that is the weight $\tau^{2}$ in the sum of Eq. (72), which emphasizes the correlators $G(\tau)$ with the largest relative errors. Caselle and Hasenbusch [23] have therefore replaced the correlation function for $\tau>\tau_{\max } \approx 3 \xi$ by its large distance form with $\xi=\xi_{\text {eff }}\left(\tau_{\max }\right)$. The sum was then extended to infinity. The procedure provides an estimate of the infinite volume value of $\mu_{2}$. On the other hand the finite size dependence of $\xi_{2 n d}$ is then lost and the determination of $\xi_{2 n d}$ is no longer independent of the other definitions. Because of that, we have derived the second moment correlation length directly from Eq. (72), truncating the sum when the numerical correlation function result was compatibel with zero. The corresponding values of $\xi_{2 n d}$ in Table 1 have relatively large errors. The values increase with increasing $L$, approaching the results from other definitions from below. At $L=120$ the estimate for $\xi_{2 n d}$ is probably too low, because of the mentioned numerical difficulties.

Our test of the different definitions of $\xi$ was performed at $T=T_{c}$. Already here we have found indications for the presence of higher states. In general, the influence of higher exitations becomes even stronger in the broken phase. An extensive discussion of this point and the actual determination of the first two higher states with a variational technique can be found in Ref. [32. In the same paper and in Ref. [23] the numerical estimates of the exponential correlation length in the broken phase have been compared to high precision results from the $Z_{2}$ gauge model 33, which is related to the Ising model by a duality transformation. The corresponding values differed at most in the third digit and showed that $\xi_{\text {eff }}$ slightly underestimates the exponential correlation length. In the high temperature phase no nearby exitations are present and $\xi_{\text {eff }}$ is a good estimator of the true correlation length. As a result of these considerations we resort in the following to the estimation of the exponential correlation length via the fit method. The corresponding scaling function can be calculated to a good approximation in this way. The extreme precision aimed at in Refs. 23] and [32] is not required for that purpose. Moreover, a possible small underestimation of $\xi$ in the broken phase can be controlled by comparing the critical amplitude ratio $\xi^{+} / \xi^{-}$, which we calculate below, to the known results for the ratio $U_{\xi_{\text {exp }}}$. 


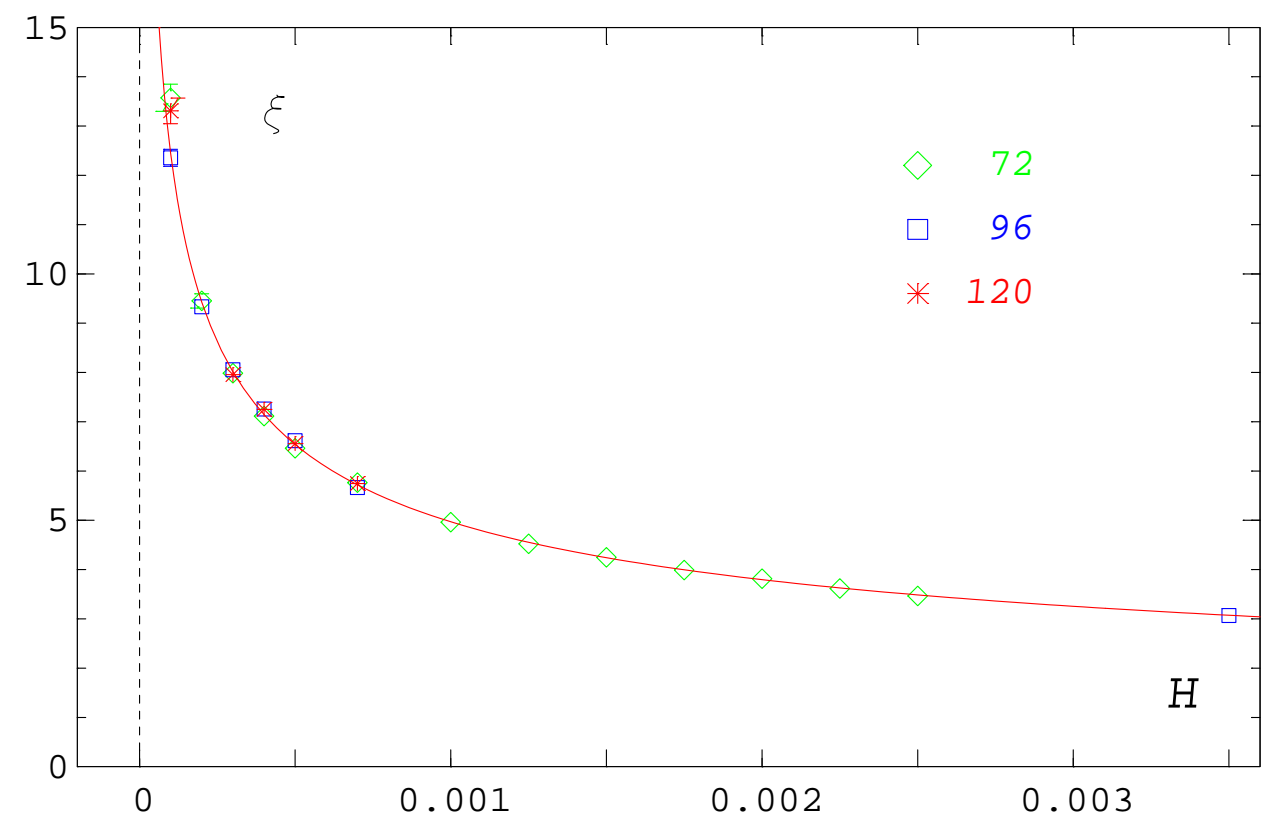

Figure 8: The correlation length $\xi$ at $T_{c}$ as a function of the magnetic field $H$ for $L=72,96$ and 120. The solid line shows the fit (76).

\subsection{The Critical Amplitudes of the Correlation Length}

We have determined the correlation length on the critical isotherm for $H>0$ on lattices with $L=72,96$ and 120. The results are shown in Fig. 8 . We have performed fits to these data with the ansatz

$$
\xi=\xi^{c} H^{-\nu_{c}}\left[1+\xi_{1}^{c} H^{\omega \nu_{c}}+\xi_{2}^{c} H\right]
$$

As in the case of the magnetization the first correction-to-scaling term turned out to be compatible with zero. Subsequently, we have therefore made fits with $\xi_{1}^{c} \equiv 0$ in the interval $0.0002 \leq H \leq 0.0035$. Irrespective of whether we use all data in the interval, or only those from the largest lattices or subsets of them we find

$$
\xi=0.3048(9) H^{-\nu_{c}}[1+9.8(2.5) H]
$$

with $\chi^{2} / N_{f}$ varying between 0.8 and 1.1 . The corresponding critical amplitude is then

$$
\xi^{c}=0.3048(9) .
$$

The correlation length data for $H=0$ below and above the critical temperature are shown in Figs. 9 (a) and (b). They are slighly more fluctuating than the data in Fig. 8. Nevertheless, the corresponding critical amplitudes may be determined quite well. Here, we have tested again that a fit ansatz without the first correction-to-scaling term is sufficient

$$
\xi=\xi^{ \pm}|t|^{-\nu}\left[1+\xi_{2}^{ \pm} t\right]
$$



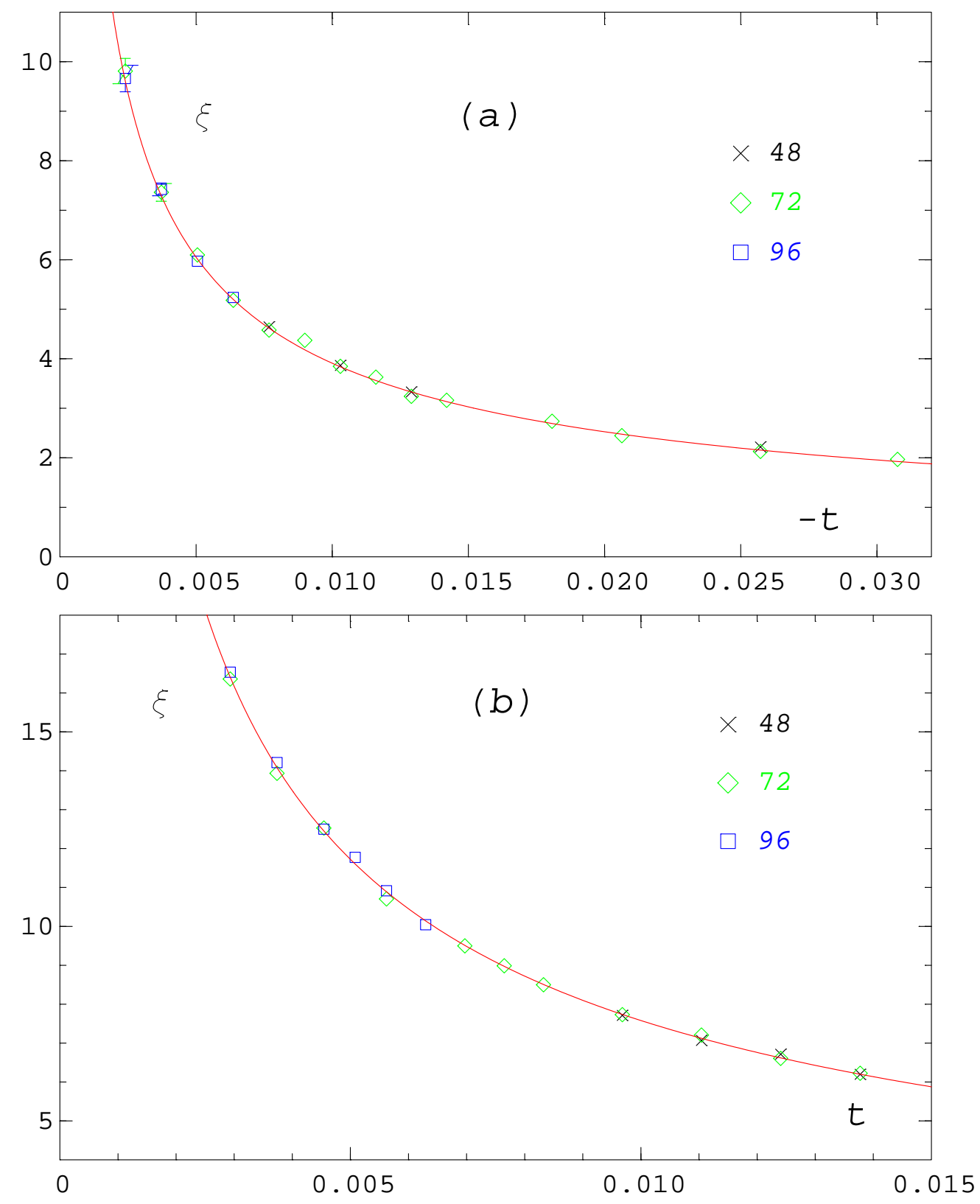

Figure 9: The correlation length $\xi$ at $H=0$ for $L=48,72$ and 96, (a) below $T_{c}$ versus $-t$ and (b) above $T_{c}$ versus $t$. The solid lines show the fits from Eqs. (79) and (81), respectively.

As it turns out, the best fits in the broken phase (with $\chi^{2} / N_{f} \approx 3$ ) are those using all points and setting $\xi_{2}^{-} \equiv 0$. We obtain

$$
\xi=0.2145(15) \cdot(-t)^{-\nu}, \quad \text { that is } \xi^{-}=0.2145(15) .
$$

The error of $\xi^{-}$includes also all other fit results, using only the data from the largest lattices and/or with a correction term proportional to $t$. In the symmetric phase the correction term is only observable, when the larger $t$-points are included in the fits. 
We have therefore first performed fits in the interval $0.002<t<0.009$ omitting the correction term and find for the critical amplitude the value

$$
\xi^{+}=0.415(1)
$$

The subsequent fit with all data leads to

$$
\xi=0.415(1) t^{-\nu}[1+0.2(1) t]
$$

with again $\chi^{2} / N_{f} \approx 3$. From the critical amplitudes for $H=0$ we derive the ratio $\xi^{+} / \xi^{-}=1.935(14)$. As discussed already, this number is to be compared to the results 1.891(22) from [23], 32, 33] and 1.896(10) from [7, 27] for this ratio. We deduce from this check, that our $\xi$-values in the broken phase are about $2 \%$ lower than the true exponential correlation length. The critical amplitudes $\xi^{+}$and $\xi^{c}$, which we have also determined, should however be unaffected by possible higher states [32]. That allows us to calculate the universal ratios $Q_{c}$ and $Q_{2}$ from Eqs. (19) and (20). We obtain

$$
Q_{c}=0.326(3) \quad \text { and } \quad Q_{2}=1.201(10)
$$

Our finding for $Q_{c}$ is well in accord with two other results: 0.328(5) from [23] and $0.324(6)$ from [26, it is slightly below the value $0.3315(10)$ from [7, 27. The number for $Q_{2}$ is the first one from MC simulations and compares well with 1.195(10) from [7, 27, the result 1.17(2) from [14, 26] is somewhat lower.

\section{$7 \quad$ The Scaling Function of the Correlation Length}

In Fig. 10] we show the data for the normalized scaling function $\hat{g}_{\xi}(z)=\xi h^{\nu_{c}} / g_{\xi}(0)$. The determination of the correlation length was sometimes difficult, in particular in the region below $T_{c}$, because the correlation function data were not always accurate enough to identify a reasonable plateau in $\xi_{\text {eff }}$. In most of these cases we could resort to the results of smaller lattices, after convincing ourselves from data adjacent in $H$, that no finite size effects were present. The data shown in Fig. 10 correspond to the ones presented in Fig. 6] for the scaling function of the susceptibility, apart from a point at $z=-10.4$. The normalization was calculated from Eq. (39) and is $g_{\xi}(0)=0.331(1)$. As in the case of the magnetization and the susceptibility we observe scaling of the correlation length data in a large $z$-range and an early approach to the asymptotic forms calculated from Eq. (40). The shape of $\hat{g}_{\xi}(z)$ is very similar to that of $f_{\chi}(z)$ - both functions have a peak at about the same $z$-value, slightly below 2. It is therefore natural to consider the ratio $\hat{g}_{\xi}^{2} / f_{\chi}$ appearing in Eq. (45). Taking the square of $\hat{g}_{\xi}$ instead of the function itself leads to a very slowly varying function of $z$. That can be seen from the asymptotic behaviour

$$
\hat{g}_{\xi}^{2} / f_{\chi}(z) \underset{z \rightarrow \pm \infty}{=} r^{ \pm}( \pm z)^{-\eta \nu}
$$




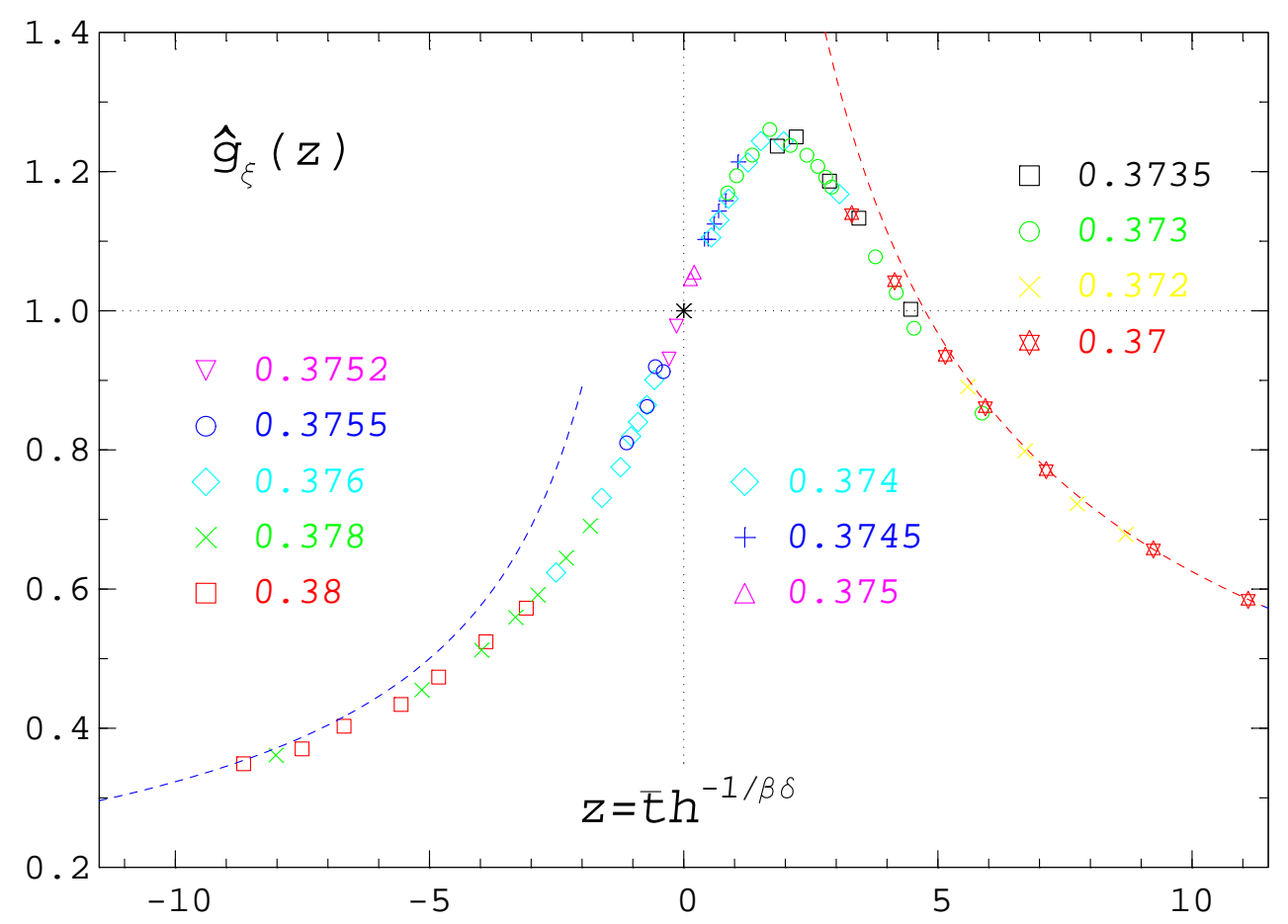

Figure 10: The scaling function of the correlation length: $\hat{g}_{\xi}(z)=\xi h^{\nu_{c}} / g_{\xi}(0)$. The dashed lines are the asymptotic forms calculated from Eq. (40). The numbers refer to the different $J=1 / T$-values of the data.

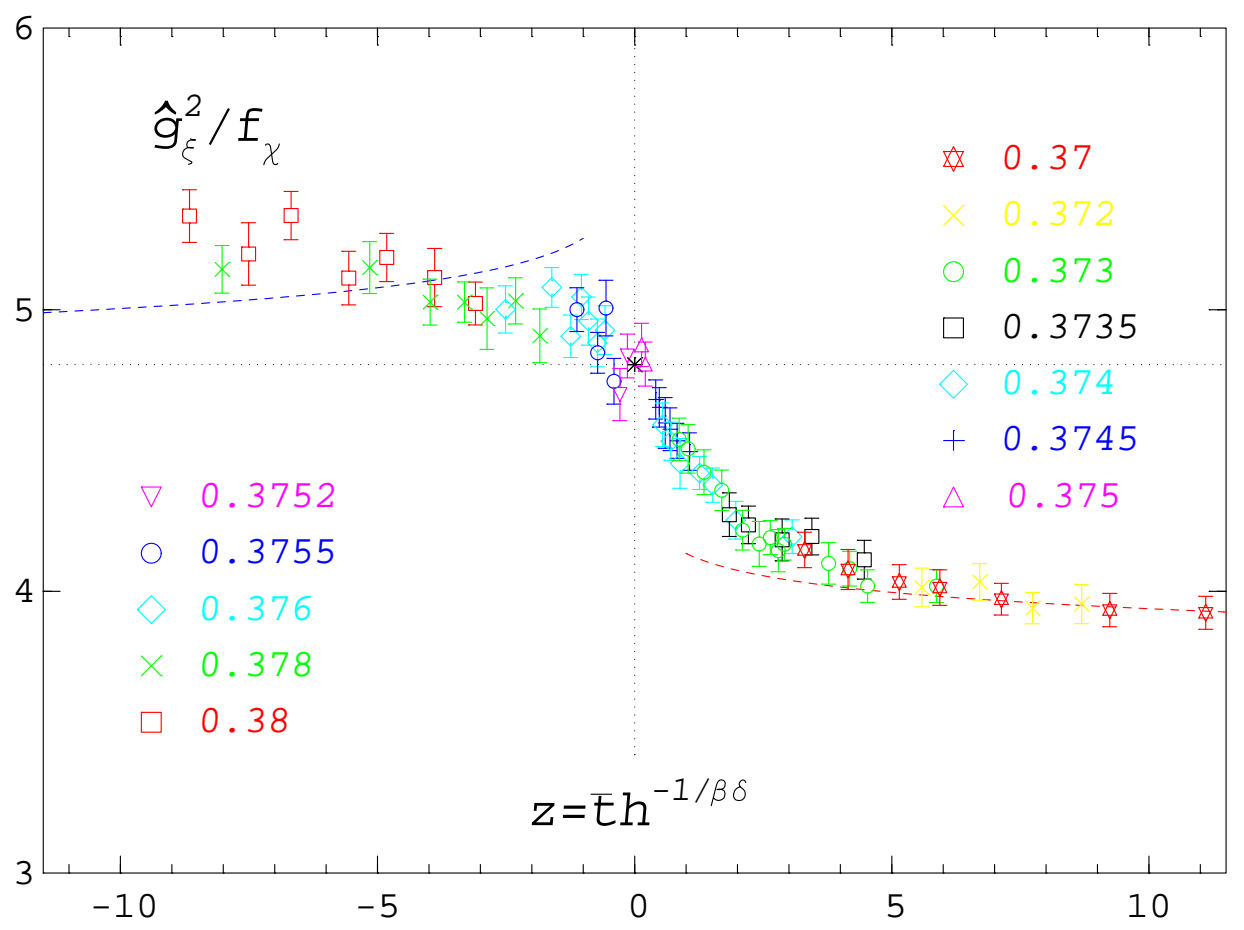

Figure 11: The ratio $\hat{g}_{\xi}^{2} / f_{\chi}(z)$ of scaling functions. The dashed lines are the asymptotic forms calculated from Eqs. (83) and (84). The numbers refer to the different $J=1 / T$-values of the data, the star shows the normalization point $\hat{g}_{\xi}^{2} / f_{\chi}(0)=\delta$. 
Here, $\eta \nu=0.0211$ and

$$
r^{+}=\left[\left(\frac{\delta}{Q_{2}}\right)^{2} R_{\chi}^{\eta}\right]^{1 /(2-\eta)} \quad \text { and } \quad \frac{r^{+}}{r^{-}}=\frac{U_{\xi}^{2}}{U_{2}}
$$

For $\eta \rightarrow 0$ one obtains an even simpler picture: the ratio $\hat{g}_{\xi}^{2} / f_{\chi}$ attains the constant values $r^{ \pm}$for $( \pm z) \rightarrow \infty$, where $r^{+}=\delta / Q_{2}=4.001$ and $r^{+} / r^{-}$is very close to $3 / 4$ (when one takes the "true" value $U_{\xi}=1.89$ ), at $z=0$ one has $\hat{g}_{\xi}^{2} / f_{\chi}=\delta=4.805$. In Fig. 11] we show the behaviour of the ratio from the actual data and we compare it to the asymptotic forms (83) derived from the amplitudes which we have determined. As expected, the ratio is slowly varying, apart from the close neighbourhood of the critical line $z=0$. In the hot phase $(z>0)$ we obviously have a consistent behaviour of the data and a clear, early approach to the asymptotic curve, whereas in the cold phase $(z<0)$ the data are more noisy, probably because of the difficulties in calculating the correlation length with sufficient precision there. We refrain here from a direct comparison with the parametric representation of Fisher et al. [6] for $\xi^{2} / 2 \chi$ because we use a slightly different set of critical exponents (those of Ref. [3]), which is crucial here. Moreover, since we consider an improved instead of the standard Ising model the relative normalizations of the variables are unclear.

\section{Summary and Conclusions}

In this paper we have calculated the equation of state for the universality class of the three-dimensional Ising model directly from Monte Carlo simulations. In addition we have determined the universal scaling functions of the susceptibility and of the correlation length. There are essentially three difficulties one encounters in such an enterprise: finite size effects, corrections to scaling and the unknown size of the critical region, where scaling works and the equation of state describes the critical behaviour of the magnetization. In order to minimize the corrections to scaling we have studied the improved Ising model as proposed in Ref. 13. We used cubic lattices of linear extension $L$ in the range 24 to 120 to prevent finite size effects and performed a large number of simulations with non-zero magnetic field $H$ at different couplings $J=1 / T$ to identify the critical region. Further simulations at $H=0$ and at $T=T_{c}$ served to determine the critical amplitudes of the magnetization, the susceptibility and the correlation length. In particular, we have calculated the amplitudes $B^{c}$ of the magnetization and $\xi^{c}$ of the correlation length for the first time from MC simulations. The critical behaviours of $M, \chi$ and $\xi$ on these lines in the $H, T$-plane could indeed be described well without the leading correction-toscaling terms. From the amplitudes, which are different from those of the standard Ising model, we have derived the universal amplitude ratios $C^{+} / C^{-}=4.756(28)$, $R_{\chi}=1.723(13), Q_{c}=0.326(3)$ and $Q_{2}=1.201(10)$, in accord with other $\mathrm{MC}$ and analytic results. Only our estimate for $U_{\xi}=1.935(14)$ differs from the corresponding 
ratio for the exponential correlation length, probably because the $\xi$-values in the broken phase were underestimated slightly due to the contribution of higher states to the correlation functions.

As it turned out, the critical region extends only to rather small values in the external field $H$, especially in the region below the critical temperature. We have compared our scaling magnetization data to the parametric representation of the equation of state by Guida and Zinn-Justin [3]-5] and find excellent agreement between theory and numerical experiment. The comparison of the susceptibility data to the corresponding scaling function shows a marginal difference in the symmetric phase, which however can be explained by the slightly different value for $R_{\chi}$ used in the parametrization. The shape of the correlation-length-scaling function is very similar to the one of the susceptibility, the peak positions of the two scaling functions are coinciding within the error bars. The ratio $\hat{g}_{\xi}^{2} / f_{\chi}$ is therefore a slowly varying function without a peak, as expected from earlier parametrizations.

\section{Acknowledgments}

We are grateful to Michele Caselle for enlightening discussions, in particular about the correlation length, and the encouragement of Tereza Mendes to start this project. We appreciate the constant interest and assistance of our colleagues Sven Holtmann and Thomas Schulze. Our work was supported by the Deutsche Forschungsgemeinschaft under Grant No. FOR 339/2-1.

\section{References}

[1] A. Pelissetto and E. Vicari, Phys. Rep. 368 (2002) 549 cond-mat/0012164.

[2] J. Zinn-Justin, Quantum Field Theory and Critical Phenomena, Clarendon Press, Oxford, 3rd Edition 1996.

[3] J. Zinn-Justin, Phys. Rept. 344 (2001) 159 hep-th/0002136.

[4] R. Guida and J. Zinn-Justin, J. Phys. A 31 (1998) 8103 cond-mat/9803240.

[5] R. Guida and J. Zinn-Justin, Nucl. Phys. B 489 (1997) 626 hep-th/9610223.

[6] M. E. Fisher, S.-Y. Zinn and P. J. Upton, Phys. Rev. B 59 (1999) 14533.

[7] M. Campostrini, A. Pelissetto, P. Rossi and E. Vicari, Phys. Rev. E 60 (1999) 3526 cond-mat/9905078.

[8] J. Engels, S. Holtmann, T. Mendes and T. Schulze, Phys. Lett. B 492 (2000) 219 hep-lat/0006023.

[9] D. Toussaint, Phys. Rev. D 55 (1997) 362 hep-lat/9607084. 
[10] J. Engels and T. Mendes, Nucl. Phys. B 572 (2000) 289 hep-lat/9911028.

[11] H. G. Ballesteros, L. A. Fernández, V. Martín-Mayor and A. Muñoz Sudupe, Phys. Lett. B 441 (1998) 330 hep-lat/9805022.

[12] M. Hasenbusch, K. Pinn and S. Vinti, Phys. Rev. B 59 (1999) 11471 hep-lat/9806012.

[13] M. Hasenbusch, J. Phys. A 32 (1999) 4851 hep-lat/9902026.

[14] S.-Y. Zinn, M. E. Fisher, Physica A 226 (1996) 168.

[15] A. L. Talapov and H. W. Blöte, J. Phys. A29 (1996) 5727 cond-mat/9603013.

[16] V. Privman, P. C. Hohenberg and A. Aharony, in Phase Transitions and Critical Phenomena, vol. 14, edited by C. Domb and J. L. Lebowitz (Academic Press, New York, 1991).

[17] B. Widom, J. Chem. Phys. 43 (1965) 3898.

[18] R. B. Griffiths, Phys. Rev. 158 (1967) 176.

[19] R. C. Brower and P. Tamayo, Phys. Rev. Lett. 62 (1989) 1087.

[20] U. Wolff, Phys. Rev. Lett. 62 (1989) 361.

[21] I. Dimitrovic, P. Hasenfratz, J. Nager and F. Niedermayer, Nucl. Phys. B 350 (1991) 893.

[22] M. Seniuch, Zustandsgleichungen und Korrelationslänge beim dreidimensionalen Isingmodell mit verbesserter Wirkung, Diploma Thesis, Universität Bielefeld, March 2002.

[23] M. Caselle and M. Hasenbusch, J. Phys. A 30 (1997) 4963 hep-lat/9701007.

[24] J. Engels and T. Scheideler, Nucl. Phys. B 539 (1999) 557 hep-lat/9808057.

[25] C. Bagnuls and C. Bervillier, Phys. Rev. E 65 (2002) 066132 hep-th/0112209.

[26] M. E. Fisher and S. Zinn, J. Phys. A 31 (1998) L629.

[27] M. Campostrini, A. Pelissetto, P. Rossi and E. Vicari, Phys. Rev. E 65 (2002) 066127 cond-mat/0201180.

[28] P. Schofield, Phys. Rev. Lett. 22 (1969) 606.

[29] P. Schofield, J. D. Lister, J. T. Ho, Phys. Rev. Lett. 23 (1969) 1098.

[30] B. D. Josephson, J. Phys. C 2 (1969) 1113. 
[31] J. Engels, V. K. Mitrjushkin and T. Neuhaus, Nucl. Phys. B 440 (1995) 555 hep-lat/9412003.

[32] M. Caselle, M. Hasenbusch and P. Provero, Nucl. Phys. B 556 (1999) 575 hep-lat/9903011.

[33] V. Agostini, G. Carlino, M. Caselle and M. Hasenbusch, Nucl. Phys. B 484 (1997) 331 hep-lat/9607029. 